\title{
Cloud Gaming: Architecture and Performance
}

\author{
Ryan Shea and Jiangchuan Liu, Simon Fraser University \\ Edith C.-H. Ngai, Uppsala University \\ Yong Cui, Tsinghua University
}

\begin{abstract}
Recent advances in cloud technology have turned the idea of cloud gaming into a reality. Cloud gaming, in its simplest form, renders an interactive gaming application remotely in the cloud and streams the scenes as a video sequence back to the player over the Internet. This is an advantage for less powerful computational devices that are otherwise incapable of running high-quality games. Such industrial pioneers as Onlive and Gaikai have seen success in the market with large user bases. In this article, we conduct a systematic analysis of state-of-the-art cloud gaming platforms, and highlight the uniqueness of their framework design. We also measure their real world performance with different types of games, for both interaction latency and streaming quality, revealing critical challenges toward the widespread deployment of cloud gaming.
\end{abstract}

hrough the utilization of elastic resources and widely deployed data centers, cloud computing has provided countless new opportunities for both new and existing applications. Existing applications, from file sharing and document synchronization to media streaming, have experienced a great leap forward in terms of system efficiency and usability through leveraging cloud computing platforms. Many of these advances have come from exploring the cloud's massive resources with computational offloading and reducing user access latencies with strategically placed cloud data centers. Recently, advances in cloud technology have expanded to allow offloading not only of traditional computation but also of such more complex tasks as high-definition $3 \mathrm{D}$ rendering, which turns the idea of cloud gaming into a reality. Cloud gaming, in its simplest form, renders an interactive gaming application remotely in the cloud and streams the scenes as a video sequence back to the player over the Internet. A cloud gaming player interacts with the application through a thin client, which is responsible for displaying the video from the cloud rendering server as well as collecting the player's commands and sending the interactions back to the cloud. Figure 1 shows a high-level architectural view of such a cloud gaming system with thin clients and cloud-based rendering.

Onlive [1] and Gaikai [2] are two industrial pioneers of cloud gaming, both having seen success with multimillionuser bases. The recent $\$ 380$ millon purchase of Gaikai by Sony [3], an industrial giant in digital entertainment and consumer electronics, shows that cloud gaming is beginning to move into the mainstream. From the perspective of industry, cloud gaming can bring immense benefits by

This research is supported by a Canadian NSERC Discovery Grant, a Swedish STINT Initial Grant, and a Chinese NSFC Major Program of International Cooperation Grant (61120106008). expanding the user base to the vast number of less powerful devices that support thin clients only, particularly smartphones and tablets. As an example, the recommended system configuration for Battlefield 3, a highly popular first-person shooter game, is a quad-core CPU, 4 Gbytes RAM, 20 Gbytes storage space, and a graphics card with at least 1 Gbyte RAM (e.g., NVIDIA GEFORCE GTX 560 or ATI RADEON 6950), which already costs more than $\$ 500$. The newest tablets (e.g., Apple's iPad with Retina display and Google's Nexus 10) cannot even meet the minimum system requirements that need a dual-core CPU over $2.4 \mathrm{GHz}$, 2 Gbytes RAM, and a graphics card with 512 Mbytes RAM, not to mention smartphones of which the hardware is limited by their smaller size and thermal control. Furthermore, mobile terminals have different hardware/software architecture from PCs (e.g., ARM rather than x86 for CPU), lower memory frequency and bandwidth, power limitations, and distinct operating systems. As such, the traditional console game model is not feasible for such devices, which in turn become targets for Gaikai and Onlive. Cloud gaming also reduces customer support costs since the computational hardware is now under the cloud gaming provider's full control, and offers better digital rights management (DRM) since the game code is not directly executed on a customer's local device.

However, cloud gaming remains in its early stage, and there remain significant theoretical and practical challenges toward its widespread deployment. In this article, we conduct a systematic analysis of state-of-the-art cloud gaming platforms, in terms of both their design and their performance. We first offer an intuitive description of the unique design considerations and challenges addressed by existing platforms. We highlight their framework design. Using Onlive as a representative, we then measure its real world performance in terms of both interaction latency and streaming quality. Finally, we discuss the future of cloud gaming as well as issues yet to be addressed. 


\begin{tabular}{lll} 
Example game type & Perspective & Delay threshold \\
\hline First person shooter (FPS) & First person & $100 \mathrm{~ms}$ \\
Role playing game (RPG) & Third person & $500 \mathrm{~ms}$ \\
Real-time strategy (RTS) & Omnipresent & $1000 \mathrm{~ms}$
\end{tabular}

Table 1. Delay tolerance in traditional gaming.

\section{Cloud Gaming: Issues and Challenges}

From low-latency live video streaming to high-performance $3 \mathrm{D}$ rendering, cloud gaming must bring together a plethora of bleeding edge technologies to function. We begin our analysis with the important design considerations currently being addressed by cloud gaming providers. A cloud gaming system must collect a player's actions, transmit them to the cloud server, process the action, render the results, encode/compress the resulting changes to the game world, and stream the video (game scenes) back to the player. To ensure interactivity, all of these serial operations must happen within milliseconds. Intuitively, this amount of time, which is defined as interaction delay, must be kept as short as possible in order to provide a rich experience to cloud game players. However, there are trade-offs: the shorter the player's tolerance for interaction delay, the less time the system has to perform such critical operations as scene rendering and video compression. Also, the lower this time threshold, the more likely a higher network latency can negatively affect a player's experience of interaction. With this is mind, we start our design discussion with delay tolerance.

\section{Interaction Delay Tolerance}

Studies on traditional gaming systems have found that different styles of games have different thresholds for maximum tolerable delay [4]. Table 1 summarizes the maximum delay that an average player can tolerate before the quality of experience (QoE) begins to degrade. As a general rule, games that are played in the first person perspective, such as the shooter game Counter Strike, become noticeably less playable when actions are delayed by as little as $100 \mathrm{~ms}$. This low delay tolerance is because such first person games tend to be actionbased, and players with a higher delay tend to have a disadvantage [5]. In particular, the outcome of definitive game changing actions such as who "pulled the trigger" first, can be extremely sensitive to the delay in an action-based first person shooter (FPS) game. Third person games, such as role playing games (RPGs), and many massively multiplayer games, such as World of Warcraft, can often have a higher delay tolerance of up to $500 \mathrm{~ms}$. This is because a player's commands in such games (e.g., use item, cast spell, or heal character) are generally executed by the player's avatar; there is often an invocation phase, such as chanting magic words before a spell is cast, and hence the player does not expect the action to be instantaneous. The actions must still be registered in a timely manner, since the player can become frustrated if the interaction delay causes them a negative outcome (e.g., they healed before an enemy attack but still died because their commands were not registered by the game in time). The last category of games are those played in an "omnipresent" view, that is, a top-down view looking at many controllable entities. Examples are real-time strategy (RTS) games like Star Craft and simulation games such as The Sims. Delays of up to $1000 \mathrm{~ms}$ can be acceptable to these styles of games since the player often controls many entities and issues many individual commands, which often take seconds or even minutes to complete. In a typical RTS game, a delay of up to $1000 \mathrm{~ms}$ for a build unit action that takes over a minute will hardly be noticed by the player.
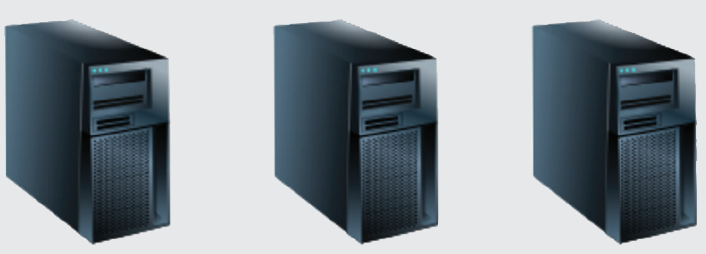

Cloud-based rendering devices

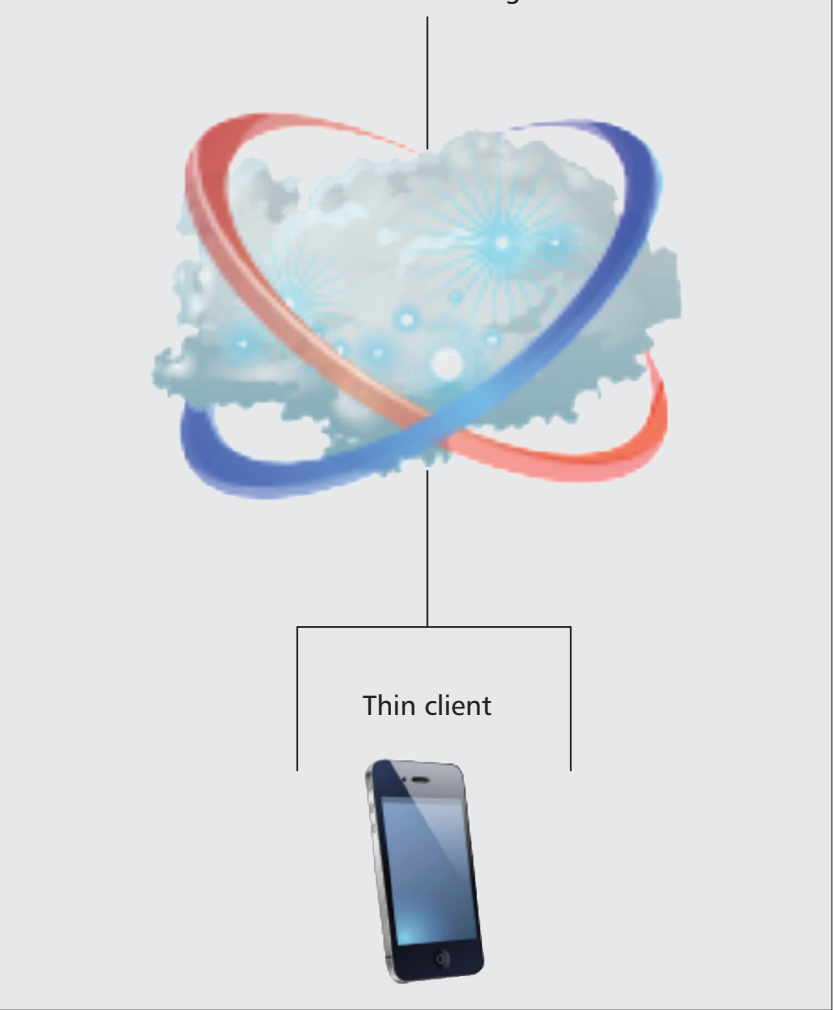

Figure 1. Cloud gaming overview.

Although there is much similarity between interaction delay tolerance for traditional gaming and cloud gaming, we must stress the following critical distinctions. First, traditionally, the interaction delay was only an issue for multiplayer online gaming systems, and was generally not considered for singleplayer games. Cloud gaming drastically changes this; now all games are being rendered remotely and streamed back to the player's thin client. As such, we must be concerned with interaction delay even for a single-player game. Also, traditional online gaming systems often hide the effects of interaction delay by rendering the action on a player's local system before it ever reaches the gaming server. For example, a player may instruct the avatar to move, and it immediately begins the movement locally; however, the gaming server may not receive the update on the position for several milliseconds. Since cloud gaming offloads its rendering to the cloud, the thin client no longer has the ability to hide the interaction delay from the player. Visual cues such as mouse cursor movement can be delayed by up to $1000 \mathrm{~ms}$, making it impractical to expect the player to be able to tolerate the same interaction delays in cloud gaming as they do in traditional gaming systems. We conjecture that the maximum interaction delay for all games hosted in a cloud gaming context should be at most $200 \mathrm{~ms}$. Other games, specifically such action-based games as FPSs, likely require less than $100 \mathrm{~ms}$ interaction delay in order not to affect the players' QoE. Recent research using subjective tests have indicated that this is indeed the case [6]. 


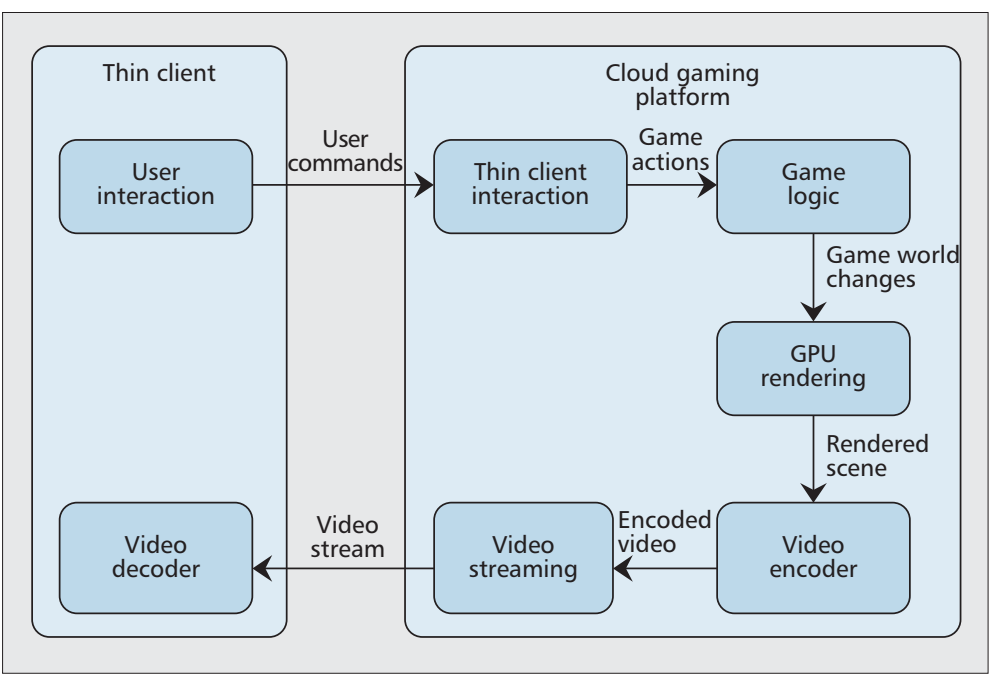

Figure 2. Framework of a cloud gaming platform.

processing unit (GPU) into a rendered scene. The rendered scene must be compressed by the video encoder and then sent to a video streaming module, which delivers the video stream back to the thin client. Finally, the thin client decodes the video and displays the video frames to the player.

To confirm the representability of this generic framework, we have conducted traffic measurement and analysis from the edge of four networks, which are located in the United States, Canada, China, and Japan. We recorded the packet flow of both Gaikai and Onlive. After that, we used Wireshark to extract packet-level details, which reveal the existence of thin clients and their interactions with remote cloud servers. We also discover that Gaikai is implemented using two public clouds: Amazon EC2 and Limelight. When a player selects a game on Gaikai, an EC2 virtual machine will first deliver the Gaikai game client to the player. After that, it forwards the IP addresses of game proxies that are ready to run

\section{Video Streaming and Encoding}

We next examine the video streaming and encoding needs of a cloud gaming system. Cloud gaming's video streaming requirements are quite similar to another classical application, live media streaming. Both cloud gaming and live media streaming must quickly encode/compress incoming video and distribute it to end users. In both, we are only concerned with a small set of the most recent video frames and do not have access to future frames before they are produced, implying encoding must be done with respect to very few frames.

However, live video streaming and cloud gaming also have important differences. First, compared to live media streaming, cloud gaming has virtually no capacity to buffer video frames on the client side. This is because when a player issues a command to the local thin client, the command must traverse the Internet to the cloud, be processed by the game logic, rendered by the processing unit, compressed by the video encoder, and streamed back to the player. Given that this must all be done in under 100-200 ms, it is apparent that there is not much margin for a buffer. Live media streaming, on the other hand, can afford a buffer of hundreds of milliseconds or even a few seconds with very little loss in the QoE of the end user.

The sensitive real-time encoding needs of cloud gaming make the choice of video encoder of paramount importance for any cloud gaming provider. Currently, the major cloud gaming providers Gaikai and Onlive both use versions of the H.264/MPEG-4 AVC encoder. Gaikai uses a software-based approach to encoding, whereas Onlive is using specialized hardware to compress its cloud gaming video streams. In either case, the choice of the H.264 encoder is motivated by the fact that the encoder not only has a very high compression ratio, but also can be configured to work well with stringent real-time demands.

\section{Cloud Gaming Framework}

Based on the design considerations we have been discussing, we now outline a generic framework for a cloud gaming system. Figure 2 shows the various functions and modules required by a cloud gaming system. As can be observed, a player's commands must be sent over the Internet from its thin client to the cloud gaming platform. Once the commands reach the cloud gaming platform, they are converted into appropriate in-game actions, which are interpreted by the game logic into changes in the game world. The game world changes are then processed by the cloud system's graphical the selected games to the players. The player will then select one game proxy to run the game. For multiplayer online games, these game proxies will also forward the players' operations to game servers and send the related information/reactions back to the players. Onlive's workflow is quite similar, but is implemented with a private cloud environment. Using public clouds enables lower implementation costs and higher scalability; however, a private cloud may offer better performance and customization that fully unleash the potential of the cloud for gaming. Hence, we use Onlive in the following measurement and analysis.

\section{Real World Performance: Onlive}

Despite some recent financial issues, Onlive was one of the first to enter the North American market and offers one of the most advanced implementations of cloud gaming available for analysis. A recent official announcement from Onlive put the number of subscribers at roughly 2.5 million, with an active user base of approximately 1.5 million. We evaluate the critically acclaimed game Batman Arkham Asylum on Onlive and compare its performance to a copy of the game running locally. In our analysis, we look at two important metrics, the interaction delay (response time) and image quality. Our hardware remains consistent for all experiments. We run Batman through an Onlive thin client as well as locally on our local test system. The test system contains an AMD 7750 dual core processor, 4 Gbytes of RAM, a 1-Tbyte 7200 RPM hard drive, and an AMD Radeon 3850 GPU. The network access is provided through a wired connection to a residential cable modem with a maximum connection speed of $25 \mathrm{Mb} / \mathrm{s}$ for download and $3 \mathrm{Mb} / \mathrm{s}$ for upload. Our system specifications and network connections exceed the recommended standards for both Onlive and the local copy of the game, which ensures that the bottleneck we will see is solely due to the intervention of the cloud.

\section{Measuring Interaction Delay}

As discussed previously, minimizing interaction delay is a fundamental design challenge for cloud gaming developers and is thus a critical metric to measure. To accurately measure interaction delay for Onlive and our local game, we use the following technique. First, we install and configure our test system with a video card tuning software, MSI afterburner. It allows users to control many aspects of the system's GPU, even the fan speed. However, we are interested in its secondary uses, particularly the ability to perform accurate screen captures of 


\begin{tabular}{lll} 
Measurement & $\begin{array}{l}\text { Processing } \\
\text { time }(\mathrm{ms})\end{array}$ & $\begin{array}{l}\text { Cloud overhead } \\
(\mathrm{ms})\end{array}$ \\
\hline Local render & 36.7 & N/A \\
Onlive base & 136.7 & 100.0 \\
Onlive $(+10 \mathrm{~ms})$ & 143.3 & 106.7 \\
Onlive $(+20 \mathrm{~ms})$ & 160.0 & 123.3 \\
Onlive $(+50 \mathrm{~ms})$ & 160.0 & 123.3 \\
Onlive $(+75 \mathrm{~ms})$ & 151.7 & 115.0
\end{tabular}

Table 2. Processing time and cloud overhead.

gaming applications. Second, we configure our screen capture software to begin recording at 100 frames/s when we press the $\mathrm{Z}$ key on the keyboard. The $\mathrm{Z}$ key also corresponds to the "Zoom Vision" action in our test game. We start the game and use the zoom vision action. By looking at the resulting video file, we can determine the interaction delay from the first frame in which our action becomes evident. Since we are recording at $100 \mathrm{frames} / \mathrm{s}$, we have a 10 -ms granularity in our measurements. To calculate the interaction delay in milliseconds, we take the frame number and multiply by $10 \mathrm{~ms}$. Since recording at 100 frames/s can be expensive in terms of CPU and hard disk overhead, we apply two optimizations to minimize the influence recording has on our games performance. First, we resize the frame to $1 / 4$ of the original image resolution. Second, we apply Motion JPEG compression before writing to the disk. These two optimizations allow us to record at 100 frames/s, while using less than 5 percent of the CPU and writing only $1 \mathrm{Mbyte} / \mathrm{s}$ to the disk.

To create network latencies, we set up a software Linux router between our test system and Internet connection. On our router we install the Linux network emulator Netem, which allows us to control such network conditions as network delay. We determine that our average baseline network round-trip time (RTT) to Onlive is approximately $30 \mathrm{~ms}$ with a 2-ms standard deviation. For each experiment we collect three samples and average them. The results can be seen in Fig. 3, where the labels on the Onlive data points indicate the added latency. For example, Onlive $(+20 \mathrm{~ms})$ indicates that we added an additional $20 \mathrm{~ms}$ on the network delay, bringing the total to $50 \mathrm{~ms}$. Our locally rendered copy has an average interaction delay of approximately $37 \mathrm{~ms}$, whereas our Onlive baseline takes approximately four times longer at $167 \mathrm{~ms}$ to register the same game action. As expected, when we simulate higher network latencies, the interaction delay increases. Impressively, the Onlive system manages to keep its interaction delay below $200 \mathrm{~ms}$ in many of our tests. This indicates that for many styles of games, Onlive could provide acceptable interaction delays. However, when the network latency exceeds $50 \mathrm{~ms}$, the interaction delays may begin to hinder the users' experience. Also, even with our baseline latency of only $30 \mathrm{~ms}$, the system could not provide an interaction delay of less than $100 \mathrm{~ms}$, the expected threshold for FPSs.

We next further break down the delay into detailed components. Returning to Fig. 3 , we define the processing time to be the amount of interaction delay caused by the game logic, GPU rendering, video encoding, etc; that is, it is the components of the interaction delay not explained by the network latency. For example, our locally rendered copy of the game has no network latency; therefore, its processing time is simply $37 \mathrm{~ms}$. Our Onlive base case, on the other hand, has its communication delayed by approximately $30 \mathrm{~ms}$ due to the network latency, meaning its processing time is approximately 137 ms. Finally, we calculate the cloud overhead, which we define to be the delay not caused by the core game logic or network latency. It includes the amount of delay caused by the video encoder and streaming system used in Onlive. To

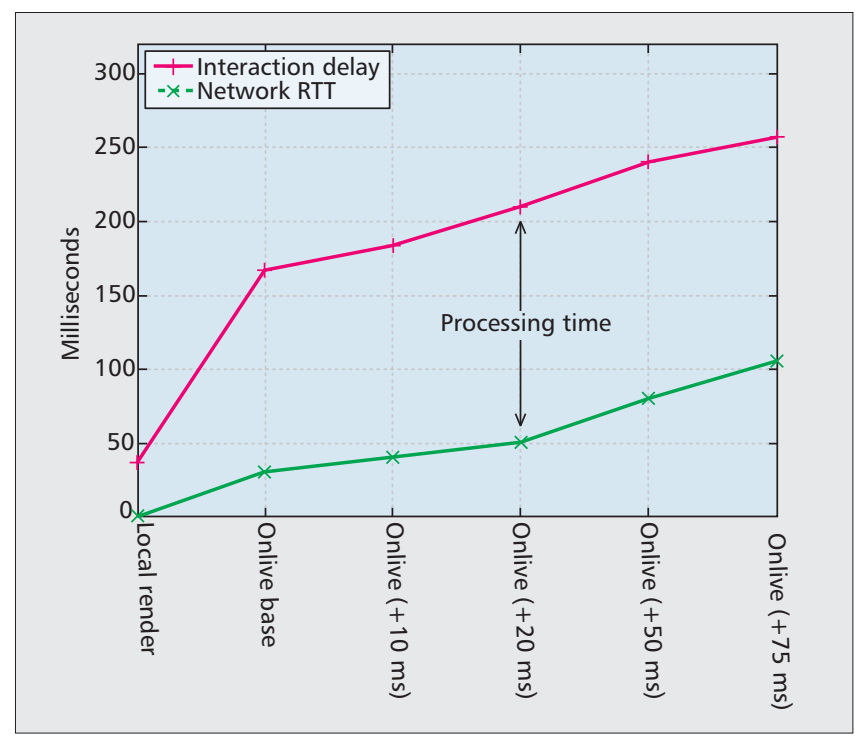

Figure 3. Interaction delay in Onlive.

calculate this number, we subtract the local render processing time of $37 \mathrm{~ms}$ from our Onlive experiment processing time. Table 2 gives the interaction processing and cloud overhead measured in our experiments. As can be seen, the cloud processing adds about $100-120 \mathrm{~ms}$ of interaction delay to the Onlive system. This finding indicates that the cloud processing overhead alone is over $100 \mathrm{~ms}$, meaning that any attempt to reach this optimal interaction delay threshold will require more efficient designs in terms of video encoders and streaming software.

\section{Measuring Image Quality}

Just as critical as low interaction delay to a cloud game player is image quality. As mentioned previously, Onlive uses a hardware H.264 encoder with a real-time encoding profile, implying the compression will cause some degree of image quality loss. Devising a methodology to objectively analyze the image quality of a commercial cloud gaming system such as Onlive has a number of technical challenges. First, to obtain an accurate sample for video quality analysis, we must be able to record a deterministic sequence of frames from Onlive and compare it to our local platform. However, although the stream is known to be encoded by H.264, the stream packets can hardly be directly captured and analyzed since it appears that Onlive is using a proprietary version of the Real Time Transport Protocol (RTP). The rendering settings used by Onlive are not publicly visible, either. For example, it remains unknown if Onlive has enabled anti-aliasing or what the draw distance is for any game. With these issues in mind, we have determined the following methodology to measure Onlive image quality.

Once again, we select the popular Batman Arkham Asylum as our test game and use the same test platform described previously. To mitigate the effect different rendering settings have on the image quality, we choose the pre-rendered intro movie of the game to record. To improve the accuracy of our analysis, we unpack the intro video's master file from the game files of our local copy of Batman Arkham Asylum. The extracted movie file has a resolution of $1280 \times 720$ pixels (720p), which perfectly matches the video streamed by Onlive. We also configured our local copy of Batman to run at $720 \mathrm{p}$. We configured our display driver to force a frame rate of 30 frames/s to match the rate of the target video. Next, we configure MSI afterburner to record the video uncompressed with a resolution of $720 \mathrm{p}$ at $30 \mathrm{frames} / \mathrm{s}$. The lack of video compression is very important as we do not want to taint the samples by applying lossy compression. 


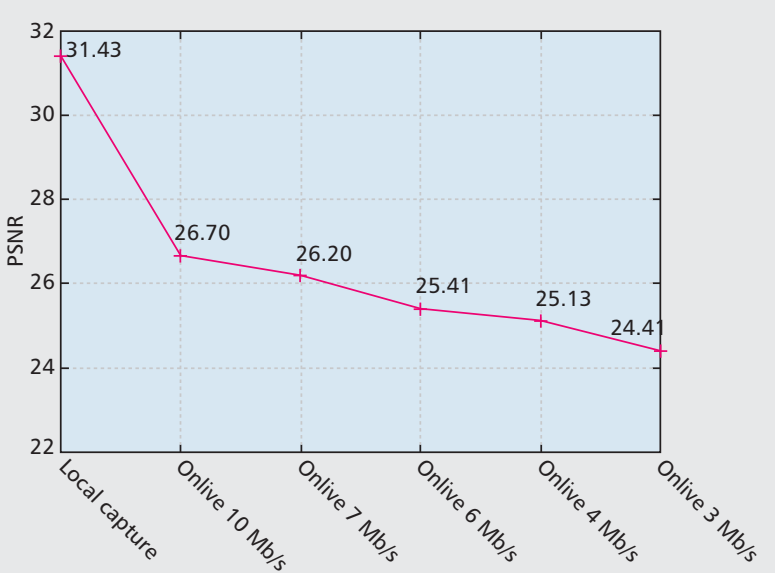

(a)

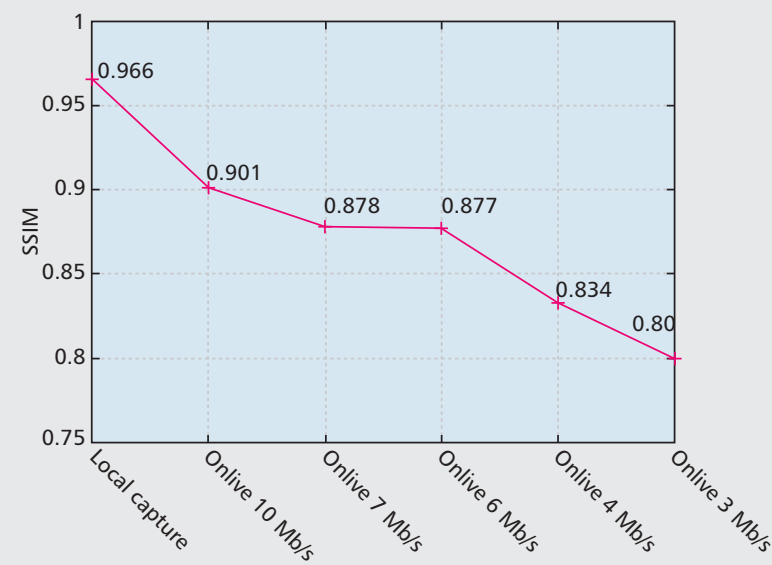

(b)

Figure 4. Onlive comparison: a) PSNR experiments; b) SSIM experiments.

We then capture the intro sequence of our locally running game and Onlive running with different bandwidth limits. To control the bandwidth, we again use our Linux software router and perform traffic shaping. We test Onlive running from its optimal bandwidth setting of $10 \mathrm{Mb} / \mathrm{s}$ gradually down to $3 \mathrm{Mb} / \mathrm{s}$. It covers a broad spectrum of bandwidths commonly available to residential Internet subscribers. Before each run, we ensure our bandwidth settings are correct by a probing test. After capturing all the required video sequences, we select the same 40-second (1200-frame) section from each video on which we will perform an image quality analysis. We analyze the video using two classical metrics: peak signal-to-moise ratio (PSNR) and structural similarity index method (SSIM). The results for PSNR are given in Fig. 4a and those for SSIM in Fig. 4b, respectively. The PSNR method quantifies the amount of error (noise) in the reconstructed video, which has been added during compression. The SSIM method calculates the structural similarity between the two video frames. As can be seen, our local capture scored a high PSNR and SSIM; however, it is not perfect, indicating some difference in the recorded video and the master file. Much of this difference is likely due to slightly different brightness and color settings used by the internal video player in the Batman game engine. When the local capture is compared to Onlive running at any connection rate, we can see a large drop in terms of both PSNR and SSIM. Since PSNR and SSIM are not on a linear scale, the drops actually indicate a considerable degradation in image quality. Generally, a PSNR of $30 \mathrm{~dB}$ and above is con- sidered good quality; however, 25 and above is considered acceptable for mobile video streaming. Not surprisingly, as we drop our test systems connection bandwidth, the image quality begins to suffer considerable degradation as well. With the exception of the $3 \mathrm{Mb} / \mathrm{s}$ test, all samples stay above a PSNR of $25 \mathrm{~dB}$; so although there is room for improvement, the image quality is still acceptable. Figure 5 illustrates the effect of Onlive's compression taken from a single frame of the opening sequence. As can be seen, the effect of compression is quite noticeable, especially as the amount of available bandwidth decreases.

\section{Conclusion and Further Discussion}

This article has closely examined the framework design of state-of-the-art cloud gaming platforms. We have also measured the performance of Onlive, one of the most representative and successful cloud gaming platforms to date. The results, particularly on interaction latency and streaming quality under diverse game, computer, and network configurations, have revealed the potential of cloud gaming as well as the critical challenges toward its widespread deployment. For future work we would like to further investigate the effect other network conditions such as packet loss and jitter have on the end user's cloud gaming experience.

Cloud gaming is a rapidly evolving technology, with many exciting possibilities. One frequently mentioned is to bring advanced $3 \mathrm{D}$ content to relatively weaker devices such as smartphones and tablets. This observation is made even more relevant by the fact that both Gaikai and Onlive are actively working on Android apps to bring their services to these mobile platforms. However, recent large-scale research indicates that it is not uncommon to find cellular network connections that have network latencies in excess of $200 \mathrm{~ms}$ [7], which alone may already cause the interaction delay to become too high for many games. Seamless integration between cellular data connection and lowerlatency WiFi connection is expected, and switching to Long Term Evolution (LTE) may help alleviate the problem. Other potential advancements involve intelligent thin clients that can perform a portion of the game rendering and logic locally to hide some of the issues associated with interaction delay, or distributed game execution across multiple specialized virtual machines [8]. This will likely require creating games specifically optimized for cloud platforms.

Besides software and service providers, hardware manufacturers have also shown a strong interest in cloud gaming, and some have begun working on dedicated hardware solutions to address the prominent issues of cloud gaming. NVIDIA has just unveiled the GeForce grid graphical processor, which is targeted specifically toward cloud gaming systems [9]. It is essentially an all-in-one graphical processor and encoding solution. The published specification shows that each of these processors has enough capability to render and encode four games simultaneously. NVIDIA's internal tests show that it can significantly mitigate the latency introduced in current cloud gaming systems [10]. It is widely expected that this type of specialized hardware will usher in a new generation of cloud gaming.

\section{References}

[1] Onlive, http://www.onlive.com/.

[2] Gaikai, http://www.gaikai.com/

[3] "Sony Buys Gaikai Cloud Gaming Service for 380 Million," Engadget, http://www.engadget.com/2012/07/02/sony-buys-gaikai/.

[4] M. Claypool and K. Claypool, "Latency and Player Actions in Online Games," Commun. ACM, vol. 49, no. 11, 2006, pp. 40-45. 
[5] M. Claypool and K. Claypool, "Latency Can Kill: Precision and Deadline in Online Games," Proc. 1st Annual ACM SIGCOMM Conf. Multimedia Sys., 2010, pp. 215-22.

[6] M. Jarschel et al., "An Evaluation of QoE in Cloud Gaming based on Subjective Tests," 5th Int'l. Conf. Innovative Mobile and Internet Services in Ubiquitous Computing, 2011, pp. 330-35.

[7] J. Sommers and P. Barford, "Cell vs. WiFi: On the Performance of Metro Area Mobile Connections," Proc. 2012 ACM Conf. Internet Measurement, 2012, pp. 301-14.

[8] Z. Zhao, K. Hwang, and J. Villeta, "Game Cloud Design with Virtualized CPU/GPU Servers and Initial Performance Results," Proc. 3rd Wksp. Scientic Cloud Computing Date, 2012 , pp. 23-30.

[9] GeForce grid, http://www.nvidia.ca/ object/grid-processors-cloud-games.html.

[10] J. Wang, "Nvidia Geforce Grid: A Glimpse at the Future of Gaming," http://www.geforce.com/whats-new/articles/geforce-grid.

\section{Biographies}

RYAN SHEA (rws1@cs.sfu.ca) received his B.Sc. degree in computer science from Simon Fraser University, Burnaby, Canada, in 2010. He is currently a Ph.D. candidate in the Network Modeling lab at Simon fraser University, where he also completed the Certificate in University Teaching and Learning. $\mathrm{He}$ is a recipient of the NSERC Alexander Graham Bell Canada Graduate Scholarship. He has worked as a network administrator with various non-profit groups. His research interests are computer and network virtualization, and performance issues in cloud computing. Recently, he received the best student paper award at IEEE/ACM IWQoS 2012 for his paper "Understanding the Impact of Denial of Service Attacks on Virtual Machines."

JIANGCHUAN LIU [S'01, M'03, SM'08] (jcliu@cs.sfu.ca) received his B.Eng. degree (cum laude) from Tsinghua University, Beiiing, China, in 1999, and his Ph.D. degree from the Hong Kong University of Science and Technology in 2003 , both in computer science. He is a recipient of a Microsoft Research Fellowship (2000), a Hong Kong Young Scientist Award (2003), and a Canada NSERC DAS Award (2009). He is a co-recipient of the IEEE ComSoc Best Paper Award on Multimedia Communications (2009), Best Paper Award of IEEE Globecom 2011, and Best Student Paper Awards of IEEE/ACM IWQoS 2008 and IWQoS 2012. He is currently an associate professor in the School of Computing Science, Simon Fraser University and was an assistant professor in the Department of Computer Science and Engineering at the Chinese University of Hong Kong from 2003 to 2004. His research interests include multimedia systems and networks, wireless ad hoc and sensor networks, and peer-to-peer and overlay networks. He is a member of Sigma Xi. He is an Associate Editor of IEEE Transactions on Multimedia, and an Editor of IEEE Communications Surveys and Tutorials. He is TPC Vice Chair for Information Systems of IEEE INFOCOM 2011.

EDITH C.-H. NGAl (edith.ngai@it.uu.se) is currently an associate professor in the Department of Information Technology, Uppsala University, Sweden. She received her Ph.D. in computer science and engineering from the Chinese University of Hong Kong in 2007. She was a post-doc at Imperial College dB, SSIM:0.89).

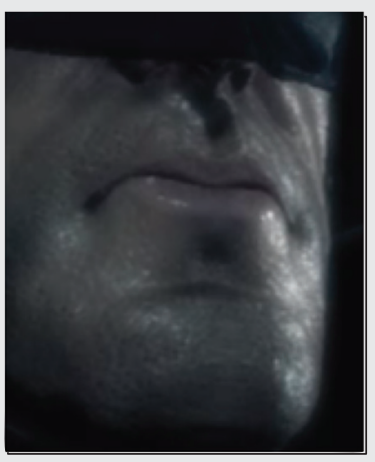

(b)

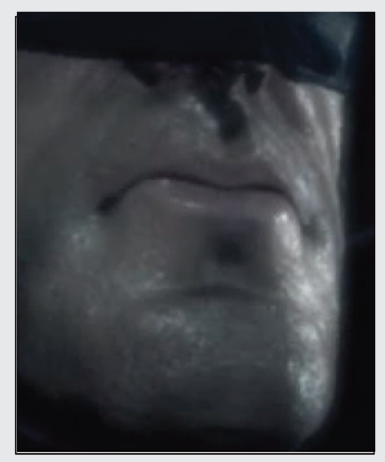

(c)

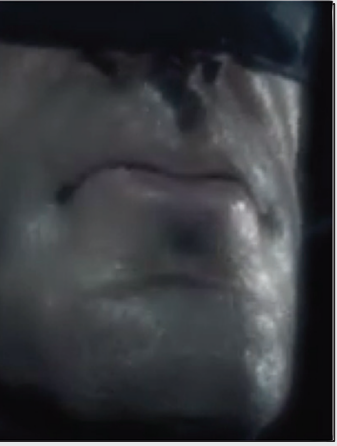

(d)

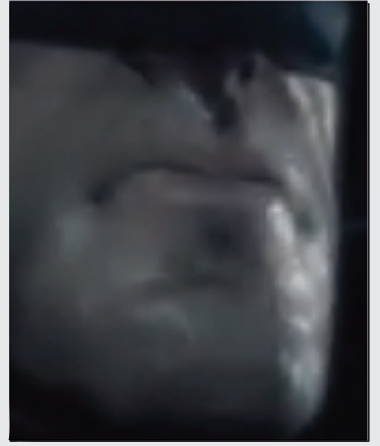

(e)
Figure 5. Image quality comparison: a) master image; b) local capture (PSNR:33.85 dB, Mb/s connection(PSNR:26.53 dB, SSIM:0.92); e) Onlive: $3 \mathrm{Mb} / \mathrm{s}$ connection (PSNR: 26.03

London in 2007-2008. Previously, she also conducted research at the University of California at Los Angeles, Simon Fraser University, and Tsinghua University. Her research areas include wireless sensor networks, mobile computing, information-centric networking, and network security and privacy. Her co-authored papers have received best paper runner-up awards at ACM/IEEE IPSN 2013 and IEEE IWQOS 2010. She served as TPC CoChair for SNCNW 2012 and Publicity Co-Chair for IEEE MSN 2012. She is a VINNMER Fellow (2009) awarded by the Swedish Governmental Agency of Innovation Systems (VINNOVA).

YONG CUI (cuiyong@tsinghua.edu.cn) received his B.E. and Ph.D. degrees from Tsinghua University, China, in 1999 and 2004, respectively. He is currently a full professor at Tsinghua University, a Council Member of the China Communication Standards Association, and Co-Chair of IETF IPv6 Transition WG Softwire. Having published more than 100 papers in refereed journals and conferences, he received the National Science and Technology Progress Award of China in 2005, the Influential Invention Award of China Information Industry in both 2012 and 2004, and best paper awards at ACM ICUIMC 2011 and WASA 2010. Holding more than 40 patents, he is one of the authors of RFC 5747 and RFC 5565 for his proposal on IPv6 transition technologies. He serves on the Editorial Boards of both IEEE TPDS and IEEE TCC. His major research interests include mobile wireless Internet and computer network architecture. 